LA-UR- $08-8042$

Approved for public release;

distribution is unilimited.

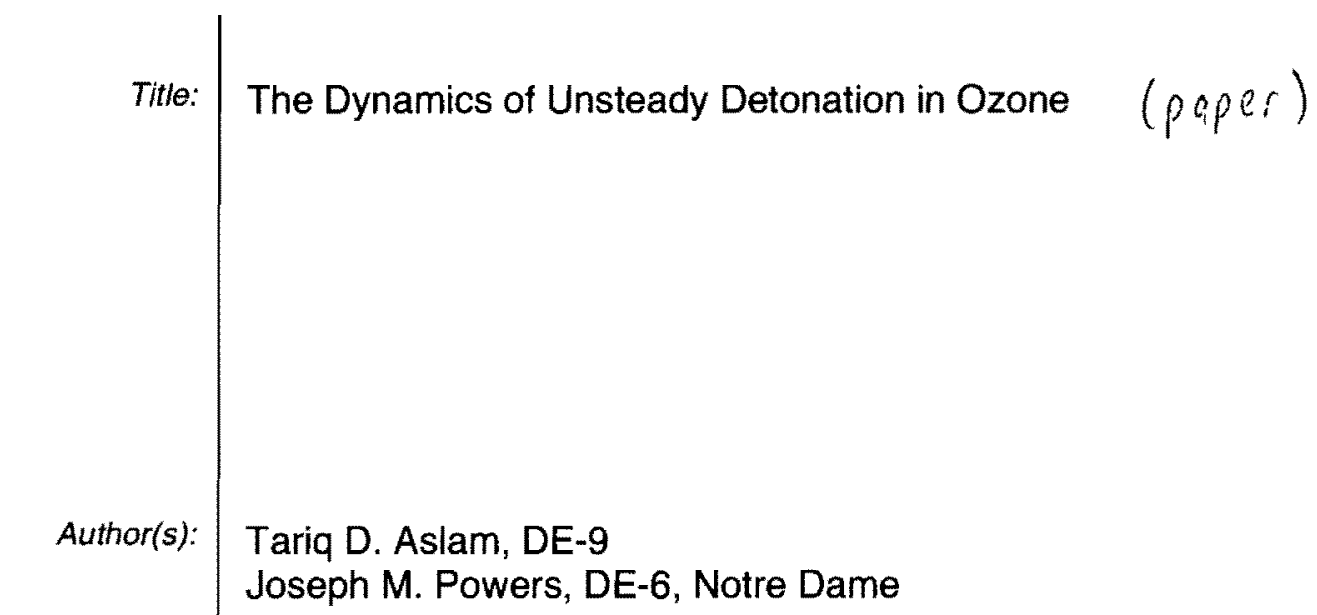

Intended for:

47th AIAA-ASM, Orlando, FL, Jan 5-8, 2009

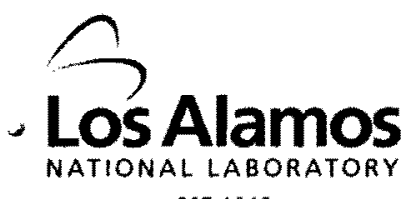

EST.1943

Los Alamos National Laboratory, an affirmative actionequal opportunity employer, is operated by the Los Alamos National Security, LLC for the National Nuclear Security Administration of the U.S. Department of Energy under contract DE-AC52-06NA25396. By acceptance of this article, the publisher recognizes that the U.S. Government retains a nonexclusive, royalty-free license to publish or reproduce the published form of this contribution, or to allow others to do so, for U.S. Government purposes. Los Alamos National Laboratory requests that the publisher identify this article as work performed under the auspices of the U.S. Department of Energy. Los Alamos National Laboratory strongly supports academic freedom and a researcher's right to publish; as an institution, however, the Laboratory does not endorse the viewpoint of a publication or guarantee its technical correctness.

Form $836(7 / 06)$ 


\title{
The Dynamics of Unsteady Detonation in Ozone
}

\author{
Tariq D. Aslam*, \\ Los Alamos National Laboratory, Los Alamos, New Mexico, 87545, USA \\ Joseph M. Powers \\ University of Notre Dame, Notre Dame, Indiana, 46556-5637, USA
}

\begin{abstract}
An ultra-fine, sub-micron discrete grid is used to capture the unsteady dynamics of a one-dimensional detonation in an inviscid $\mathrm{O}-\mathrm{O}_{2}-\mathrm{O}_{3}$ mixture. The ultra-fine grid is necessary to capture the length scales revealed by a complementary analysis of the steady detonation wave structure. For the unsteady calculations, shock-fitting coupled with a high order spatio-temporal discretization scheme combine to render numerical corruption negligible. As a result, mathematically verified solutions for a mixture initially of all $\mathrm{O}_{3}$ at one atmosphere and $298.15 \mathrm{~K}$ have been obtained; the solutions are converging at a rate much faster than the sub-first order convergence rate of all shock-capturing schemes. Additionally, the model has been validated against limited experimental data. Transient calculations show that strongly overdriven waves are stable and moderately overdriven waves unstable. New limit cycle behavior is revealed, and the first high resolution bifurcation diagram for detonation with detailed kinetics is found.
\end{abstract}

\section{Introduction}

$W^{E}$ significantly extend calculations of unsteady detonation in ozone mixtures first reported by the authors $^{1}$ to capture long time limit cycle behavior and bifurcation phenomena. Much of the discussion of the original work is repeated for completeness; the calculations and results are new for the present study. Recently, a shock-fitting method ${ }^{2}$ coupled with a high order spatial discretization ${ }^{3}$ was used to fully capture the unsteady dynamics of an inviscid one-dimensional detonation for the case of pulsating detonations which obey simple one step kinetics. ${ }^{2}$ The high accuracy of the method enabled the prediction of a rich set of bifurcation phenomena, including a transition to chaos at a rate consistent with the geometric progression characterized by Feigenbaum's constant. Formal grid convergence studies revealed that the error was converging at a rate approaching the fifth order error of the spatio-temporal discretization employed. The key to obtaining such results was the use of a shock-fitting technique so as to avoid the corrupting influences of common shock-capturing methods. Convergence rates for shock-capturing methods are at or below first order, even if nominally high order methods are used in smooth regions of the flow. ${ }^{3}$

Here, we extend this approach by using a more realistic detailed kinetics model, which introduces additional length scales, relative to the one-step model. With an analysis of spatial eigenvalues, Powers and Paolucci ${ }^{4}$ have shown for inviscid hydrogen-air detonations that the length scales for steady ChapmanJouguet $(C J)$ detonations can span over five orders of magnitude: near equilibrium, the smallest length scale is $\sim 10^{-5} \mathrm{~cm}$ and the largest is $\sim 10^{0} \mathrm{~cm}$; away from equilibrium, the breadth can be larger. Resolving this wide range of scales, necessary for a mathematically verified and scientifically repeatable calculation, poses a daunting challenge. Adaptive methods ${ }^{5,6}$ can be used effectively when extended to the viscous regime, but for inviscid flows, there remain fundamental difficulties in formally adapting a grid to a shock discontinuity.

The fine reaction scales can be attributed to the fact that constitutive laws for detailed kinetics models are a manifestation of an averaged representation of a molecular collision model in which the fundamental length

\footnotetext{
*Technical Staff Member, Dynamic and Energetic Materials Division, AIAA Member, aslam@lanl.gov.

†Professor, Department of Aerospace and Mechanical Engineering, AIAA Associate Fellow, powers@nd.edu.

Copyright (C) 2009 by Joseph M. Powers. Published by the American Institute of Aeronautics and Astronautics, Inc. with permission.
} 
scale is the mean free path. ${ }^{7,8}$ This suggests that collision-based mass, momentum, and energy diffusion, which has a role at such fine scales, should be modeled as well. However, we choose here to defer such calculations to the future, and in this paper introduce only resolved detailed kinetics coupled with advective transport. Certainly, there is an extensive literature (cf. Fedkiw, et al. ${ }^{9}$ Oran, et al., ${ }^{10} \mathrm{Hu}$, et al. ${ }^{11}$ Wang, et al. ${ }^{12}$ Walter and da Silva, ${ }^{13}$ He and Karagozian, ${ }^{14}$ or Tsuboi, et al. ${ }^{15}$ ) which makes the same inviscid assumption. Notably, Tsuboi, et al, in their recent calculations that required significant super-computer resources, employ grids sizes near $10^{-4} \mathrm{~cm}$ for their three-dimensional unsteady calculations of detonations in hydrogen-air, and report strong sensitivity of wave dynamics to the fineness of the grid. Consequently, we believe assessment of the common inviscid approach, stripped here from the method-dependent distortions of numerical diffusion, is a useful exercise, even if one ultimately wants to consider models with more physical richness.

In this paper, we consider an ozone-based mixture, $\mathrm{O}-\mathrm{O}_{2}-\mathrm{O}_{3}$, in which, relative to hydrogen-air, there are fewer reactions, and the range of length scales is smaller $\left(\sim 10^{5}\right.$ for hydrogen-air, $\sim 10^{3}$ for ozone). This reduction of the number of reactions, and more importantly, the range of scales, enables the resolved computation to be completed in a reasonable time. Kinetics for this system are well characterized ${ }^{16-18}$ and limited experimental data for ozone detonation are available. ${ }^{19}$ We give a brief synopsis of the mathematical model and computational method. This is followed by a validation of the model by comparison of the predictions of steady state detonation waves with experimental data. We then perform unsteady simulations at a variety of overdrives. Strong overdrive yields a stable solution which converges to the independent predictions of the steady wave model. As overdrive is weakened, the steady solution loses its stability, and the solution undergoes a bifurcation process in which the long term behavior is described by non-linear limit cycles. This bifurcation process is qualitatively similar to that predicted from one-step kinetics models. ${ }^{2}$ We close with brief conclusions.

\section{Mathematical Model}

The governing equations are the one-dimensional reacting Euler equations for a mixture of calorically imperfect ideal gases which react via mass action kinetics. They are of the form

$$
\frac{\partial q_{i}}{\partial t}+\frac{\partial f_{i}\left(q_{j}\right)}{\partial x}=g_{i}\left(q_{j}\right)
$$

where Eq. (1) represents the conservation of mass, linear momentum, and energy, as well as the evolution of molecular species. These are supplemented by a standard set of constitutive equations to complete the system. Full details of the model equations have been previously published. ${ }^{4}$ Here $q_{i}$ is a vector of state variables, $f_{i}$ is a flux of the state variables, and $g_{i}$ is a reaction source, also a function of the state variables. Time is $t$, and $x$ is the distance coordinate. Equation (1) is valid in smooth regions of the flow, and represents a set of hyperbolic partial differential equations. The set admits discontinuities propagating at speed $D$. Equation (1) is not valid across such a discontinuity and must be supplemented by a set of Rankine-Hugoniot jump conditions, which take the form

$$
D(t)=\frac{f_{i}^{s}-f_{i}^{o}}{q_{i}^{s}-q_{i}{ }^{o}}
$$

where $D$ is the time-dependent shock speed, $s$ denotes the shocked state, and $o$ the unshocked state.

We employ a three step, three species $\left(\mathrm{O}, \mathrm{O}_{2}, \mathrm{O}_{3}\right)$ model. The reaction mechanism is that of Hirschfelder, et $a .^{16}$ as used later by Margolis ${ }^{17}$ and Singh, et al., ${ }^{18}$ each of whom provide numerical values of rate constants.

$$
\begin{aligned}
O_{3}+M & \leftrightarrows O_{2}+O+M \\
O+O_{3} & \leftrightarrows 2 O_{2} \\
O_{2}+M & \leftrightarrows 2 O+M
\end{aligned}
$$

Here $M$ represents an inert third body, taken to have a collision efficiency of unity.

\section{Computational Method}

For spatially dependent steady wave calculations, a double precision FORTRAN-77 code has been developed and linked with the International Mathematical and Statistical Libraries (IMSL) routines DFDJAC for Jacobian 
evaluation, DEVLRG for eigenvalue estimation, a double precision version of the public domain edition of the CHEMKIN package ${ }^{20,21}$ to obtain kinetic rates and thermodynamics properties, and the standard LSODE ${ }^{22}$ solver to yield highly accurate solutions to the governing ordinary differential equations. Typical run times on a Linux-based desktop computer were ten seconds.

For transient calculations, the shock-fitting strategy of Henrick et al. ${ }^{2}$ has been adapted to allow for an arbitrary number of chemical species as well as general equations of state, suitable to interface with the CHEMKIN package. The underlying numerical scheme is a method of lines with fifth order upwind-central spatial and fifth order Runge-Kutta temporal discretization. Special one-sided differences were utilized near the shock, as well as a shock state being enforced as a boundary condition. Typical run times on Macintosh desktop computer were two hours.

\section{Results}

Here, we report a series calculations for ozone detonations with an initial mass fraction composition of $Y_{O_{3}}=1, Y_{O_{2}}=0, Y_{O}=0$ at $298.15 \mathrm{~K}$ and $1.01325 \times 10^{6}$ dyne $/ \mathrm{cm}^{2}$.

\section{IV.A. Validation for steady near- $C J$ wave}

For such a system, Streng, et al. ${ }^{19}$ report an observed $C J$ velocity of $1.863 \times 10^{5} \pm 2 \times 10^{3} \mathrm{~cm} / \mathrm{s}$. From equilibrium thermochemistry using no kinetics, they calculated a equilibrium temperature, equilibrium pressure, and detonation wave speed of $3340 \mathrm{~K}, 3.1188 \times 10^{7} \mathrm{dyne} / \mathrm{cm}^{2}$, and $1.878 \times 10^{5} \mathrm{~cm} / \mathrm{s}$, respectively.

Using the method of Powers and Paolucci, ${ }^{4}$ for steady waves, one can employ conservation of mass, momentum, energy, atomic elements to reduce the system to two ordinary differential equations. These are solved from the shock state to the equilibrium end state. This results in an equilibrium temperature, equilibrium pressure, and detonation wave speed of $3571.4 \mathrm{~K}, 3.4111 \times 10^{7} \mathrm{dyne} / \mathrm{cm}^{2}$, and $1.936555 \times$ $10^{5} \mathrm{~cm} / \mathrm{s}$, respectively. Our mixture required a detonation velocity slightly above the $C J$ velocity, so as to avoid sonic singularities within the reaction zone. This slight overdrive, as well as uncertainties in the equations of state used in the study of Streng, et al. likely explain the small discrepancies.

\section{IV.B. Stable Strongly Overdriven}

We next consider a strongly overdriven case in which the overdrive is such that the steady detonation speed is $2.5 \times 10^{5} \mathrm{~cm} / \mathrm{s}$.

\section{IV.B.1. Steady Structure}

Again with the method of Powers and Paolucci, ${ }^{4}$ the spatial distribution of dependent variables can be calculated; additionally, the spatial eigenvalues of the local Jacobian matrix can be found, and local length scales predicted. Because the system can be reduced to two coupled ordinary differential equations, there are two fundamental length scales in play. These length scales are attributable to the combined effects of advection and reaction. The length scale analysis reveals that in the near post-shock zone of the detonation, the fine and coarse scales are $6.4 \times 10^{-8}$ and $2.7 \times 10^{-7} \mathrm{~cm}$, respectively. Near equilibrium, the fine and coarse scales are $2.9 \times 10^{-7}$ and $5.8 \times 10^{-4} \mathrm{~cm}$, respectively. Figure 1 shows a plot of the evolution of the magnitudes of the resulting two length scales of the system. The two length scales coincide near $x=10^{-6} \mathrm{~cm}$. This is due to the spatial eigenvalues becoming complex conjugate pairs, with equal real parts, in this thin zone. The length scales here were based on the real part of the eigenvalues only. One could easily adjust the figure in this zone to include the length scale of oscillation, manifested in the imaginary component of the eigenvalue; this would have limited value.

In the zone extremely near the shock, $x<10^{-8} \mathrm{~cm}$, the continuum model predicts power law growth of the $\mathrm{O}$ and $\mathrm{O}_{2}$ species mass fraction, as seen in Figure 2. This growth modulates at the beginning of the induction zone, near $x=5 \times 10^{-8} \mathrm{~cm}$, which correlates well with the finest length scale in this region. The induction zone terminates near $x=1.8 \times 10^{-7} \mathrm{~cm}$. At this point, vigorous reaction commences. The system first relaxes to a partial equilibrium at $x=4 \times 10^{-6} \mathrm{~cm}$, stays on a plateau for a few decades, and relaxes to final equilibrium near $x=10^{-3} \mathrm{~cm}$. The global length scale is well estimated by the longest length scale predicted by the spatial eigenvalue analysis. 
Plots of temperature and pressure versus distance are shown in Figures 3 and 4, respectively. Here the two disparate length scales are most clearly manifested in the initial relaxation process commencing near $x=10^{-7} \mathrm{~cm}$ and the final relaxation commencing near $x=10^{-4} \mathrm{~cm}$. It appears that the main temperature rise around $x=10^{-7} \mathrm{~cm}$ is attributable to $O_{3}$ converting to $O_{2}$ over the short length scale. All species then are in a partial equilibrium over several decades of length until a final, smaller temperature drop occurs around $x=10^{-4} \mathrm{~cm}$, which close examination reveals may be due to $O_{3}$ and $O$ coming into final equilibrium. The final temperature and pressure are $4285.8 \mathrm{~K}$ and $9.2554 \times 10^{7} \mathrm{dyne} / \mathrm{cm}^{2}$, respectively.

It is lastly noted that in all plots for clarity, we give predictions, formally admitted by the continuum model, down to length scales slightly below $10^{-9} \mathrm{~cm}$. Of course, the continuum model is only physically valid down to length scales at or above the mean free path scale, which for this system can be roughly estimated to be $\sim 10^{-7} \mathrm{~cm}$. This correlates well with the finest length scale prediction from the spatial eigenvalue analysis. One can argue, with some justification, that at such fine length scales, one should account for physical diffusive transport. Here, we simply note that there is a long history of solving reactive Euler equations with detailed kinetics, and that there is value in seeing what such models predict when the distortion of numerical diffusion is rendered small by use of fine grids.

\section{IV.B.2. Transient Behavior}

Performing time-dependent shock-fitted computations on this strongly overdriven detonation reveals that the one-dimensional detonation is stable. The calculation was initialized with the highly resolved steady solution of the previous section. Figure 5 shows the computed detonation velocity versus time for three spatial resolutions. For this stable case, the steady solution is the exact solution for all time; any differences between the steady solution and that obtained with the transient code are consequences of the slightly coarser (but still ultra-fine) grid used in the transient calculation. This can also serve as a test problem for verification of the numerical scheme. It is demonstrated in Figure 5 that under resolution, the solution tends towards the exact solution of $D(t)=2.5 \times 10^{5} \mathrm{~cm} / \mathrm{s}$. Furthermore, the solution is very accurate; relative percent errors in the detonation velocity at $t=10^{-9} \mathrm{~s}$ are, from lowest to highest resolution, $0.44 \%, 0.1 \%$ and $0.012 \%$. The grids employed had spatial resolutions of $\Delta x=1 \times 10^{-7}, 5 \times 10^{-8}$, and $2.5 \times 10^{-8} \mathrm{~cm}$, with corresponding number of grid points of $1 \times 10^{4}, 2 \times 10^{4}$, and $4 \times 10^{4}$. The domain length was $10^{-3} \mathrm{~cm}$. Although these errors are diminishing superlinearly, the point of asymptotic convergence in error has yet to be reached.

\section{IV.C. Unstable Moderately Overdriven}

New calculation reveals the neutral stability boundary to be $D=2.425 \times 10^{5} \mathrm{~cm} / \mathrm{s} \pm 25 \times 10^{2} \mathrm{~cm} / \mathrm{s}$. See Figure 6 for the time dependent detonation velocity for three different initial overdrives. For $D=2.45 \times 10^{5} \mathrm{~cm} / \mathrm{s}$ and $D=2.5 \times 10^{5} \mathrm{~cm} / \mathrm{s}$, the detonation structure is stable, while for $D=2.4 \times 10^{5} \mathrm{~cm} / \mathrm{s}$, the structure is unstable. For detonations with overdrives corresponding to $2.1 \times 10^{5} \mathrm{~cm} / \mathrm{s}<D<2.45 \times 10^{5} \mathrm{~cm} / \mathrm{s}$, pulsating detonations are observed at times long compared to the pulsation frequency. See Figure 7 for the late time behavior of $D$ for an initial overdrive corresponding to $D=2.4 \times 10^{5} \mathrm{~cm} / \mathrm{s}$. For initial overdrives corresponding to $D<2.1 \times 10^{5} \mathrm{~cm} / \mathrm{s}$, the oscillation amplitude is of sufficient strength $(T>6000 \mathrm{~K})$ to cause the curve fits used for thermodynamic properties to be outside their realm of validity. Figure 8 shows a bifurcation diagram of the long time dynamics of the problem as a function of wave speed (Tariq-improve the writing here, as well as improve the figure!).

As was previously demonstrated, ${ }^{1}$ there is a severe disparity in scales within realistic gaseous detonation reaction zones. For the present ozone model, the disparity in length scales is $\mathrm{O}\left(10^{4}\right)$. Thus, one needs $10^{4}-10^{6}$ computational spatial cells and $10^{5}-10^{7}$ time integration steps, or equivalently $10^{9}-10^{13}$ computational cell updates. These simulations, on a modern single processor, will take $O$ (hour)-O(year). In dimensional terms, these detonations oscillate with a period of $1.3 \times 10^{-10} \mathrm{~s}$ at a wavelength of $3.2 \times 10^{-5} \mathrm{~cm}$. Simple calculation shows these scales are above the scales of molecular collisions and in the continuum regime for this mixture. Nevertheless, the fineness of these continuum length and time scales present a serious challenge for computational modeling, and demand a resolution far finer than that which is commonly used. 


\section{Conclusions}

It has been demonstrated that mathematically verified unsteady detonation dynamics can be predicted when sub-micron structures, admitted by continuum detailed kinetics models, are captured with ultra-fine grids. Furthermore, shock-fitting coupled with high order spatio-temporal discretization assures negligible numerical corruption of solutions to the underlying partial differential equations. Predicted detonation dynamics for the detailed kinetics of ozone are qualitatively similar to previously studied one-step models; ${ }^{2}$ notably a similar bifurcation pattern for long time limit cycle amplitudes is predicted. It is also noted that at these fine length scales, which are comparable to molecular mean free paths, diffusion will play a role and should be included in future work.

\section{Acknowledgments}

This work was performed under the auspices of the United States Department of Energy.

\section{References}

\footnotetext{
${ }^{1}$ Aslam, T. D., and Powers, J. M., "Shock-Fitted Calculation of Unsteady Detonation in Ozone," 46th AIAA Aerospace Science Meeting and Exhibit, ALAA 2008-1036, Reno, Nevada, 2008.

${ }^{2}$ Henrick, A. K., Aslam, T. D., and Powers, J. M., "Simulations of Pulsating One-Dimensional Detonations with True Fifth Order Accuracy," Journal of Computational Physics, Vol. 213, No. 1, 2006, pp. 311-329.

${ }^{3}$ Henrick, A. K., Aslam, T. D., and Powers, J. M., "Mapped Weighted Essentially Non-Oscillatory Schemes: Achieving Optimal Order Near Critical Points," Journal of Computational Physics, Vol. 207, No. 2, 2005, pp. 542-567.

${ }_{4}^{4}$ Powers, J. M., and Paolucci, S., "Accurate Spatial Resolution Estimates for Reactive Supersonic Flow with Detailed Chemistry," AIAA Joumal, Vol. 43, No. 5, 2005, pp. 1088-1099.

${ }^{5}$ Singh, S., Rastigejev, Y., Paolucci, S., and Powers, J. M., "Viscous Detonation in $\mathrm{H}_{2}-\mathrm{O}_{2}-\mathrm{Ar}$ Using Intrinsic LowDimensional Manifolds and Wavelet Adaptive Multilevel Representation," Combustion Theory and Modelling, Vol. 5, No. 2, 2001, pp. 163-184.

"Yuan, L., and Tang, T., "Resolving the Shock-Induced Combustion by an Adaptive Mesh Redistribution Method," Journal of Computational Physics, Vol. 224, No. 2, 2007, pp. 587-600.

${ }^{7}$ Powers, J. M., and Paolucci, S., "Accurate Estimates of Fine Scale Reaction Zone Thicknesses in Hydrocarbon Detonations," 44th AIAA Aerospace Sciences Meeting and Exhibit, Reno, Nevada, AIAA-2006-0950, 2006.

${ }^{8}$ Al-Khateeb, A. N., Powers, J. M., and Paolucci, S., "Length Scale Issues in Combustion Modelling," Combustion Theory and Modelling, in review, 2008.

${ }^{9}$ Fedkiw, R. P., Merriman, B., and Osher, S., "High Accuracy Numerical Methods for Thermally Perfect Gas Flows with Chemistry," Journal of Computational Physics, Vol. 132, No. 2, 1997, pp. 175-190.

${ }^{10}$ Oran, E. S., Weber, J. W., Stefaniw, E. I., Lefebvre, M. H., and Anderson, J. D., "A Numerical Study of a TwoDimensional $\mathrm{H}_{2}-\mathrm{O}_{2}-\mathrm{Ar}$ Detonation Using a Detailed Chemical Reaction Model," Combustion and Flame, Vol. 113, Nos. 1-2, 1998 , pp. 147-163.

${ }^{11} \mathrm{Hu}, \mathrm{X}$. Y., Khoo, B. C., Zhang, D. L., and Jiang, Z. L., "The Cellular Structure of a Two-Dimensional $\mathrm{H}_{2} / \mathrm{O}_{2} / \mathrm{Ar}$ Detonation Wave," Combustion Theory and Modeling, Vol. 8, No. 2, 2004, pp. 339-359.

${ }^{12}$ Wang, B., He, H., and Yu, S. T. J., "Direct Calculation of Wave Implosion for Detonation Initiation," AIAA Joumal, Vol. 43, No. 10, 2005, pp. 2157-2169.

${ }^{13}$ Walter, M. A. T., and da Silva, L. F. F., "Numerical Study of Detonation Stabilization by Finite Length Wedges," $A I A A$ Journal, Vol. 44, No. 2, 2006, pp. 353-361.

${ }^{14} \mathrm{He}, \mathrm{X}$, and Karagozian, A. R., "Pulse-Detonation-Engine Simulations with Alternative Geometries and Reaction Kinetics," Journal of Propulsion and Power, Vol. 22, No. 4, 2006, pp. 852-861.

${ }^{15}$ Tsuboi, N., Eto, K., and Hayashi, A. K., "Detailed Structure of Spinning Detonation in a Circular Tube," Combustion and Flame, Vol. 149, Nos. 1-2, 2007, pp. 144-161.

${ }^{16}$ Hirschfelder, J. O., Curtiss, C. F., and Campbell, D. E,, "The Theory of Flame Propagation. IV," Journal of Physical Chemistry, Vol. 57 , No. 4, 1953, pp. 403-414.

${ }^{17}$ Margolis, S. B.s "Time-Dependent Solution of a Premixed Laminar Flame," Journal of Computational Physics, Vol. 27, No. 3,1978 , pp. $410-427$.

${ }^{18}$ Singh, S., Powers, J. M., and Paolucci, S., "On Slow Manifolds of Chemically Reactive Systems," Journal of Chemical Physics, Vol. 117, No. 4, 2002, pp. 1482-1496.

${ }^{19}$ Streng, A. G., Stokes, C. S., and Streng, L. A., "Detonation Velocity of Gaseous Ozone," Journal of Chemical Physics, Vol. 29 , No. 2,1958 , pp. $458-459$.

${ }^{20}$ Kee, R. J., Rupley, F. M., and Miller, J. A., "Chemkin-II: A Fortran Chemical Kinetics Package for the Analysis of Gas Phase Chemical Kinetics," Sandia National Labs., Rept. SAND89-8009B, Livermore, CA, 1992.

${ }^{21}$ Kee, R. J., Rupley, F. M., and Miller, J. A., "The Chemkin Thermodynamic Data Base," Sandia National Labs., Rept. SAND87-8215B, Livermore, CA, 1992.

${ }^{22}$ Hindmarsh, A. C., "ODEPACK, a Systematized Collection of ODE Solvers," Scientific Computing, edited by R. S. Stepleman, et al., North-Holland, Amsterdam, 1983, pp. 55-64.
} 


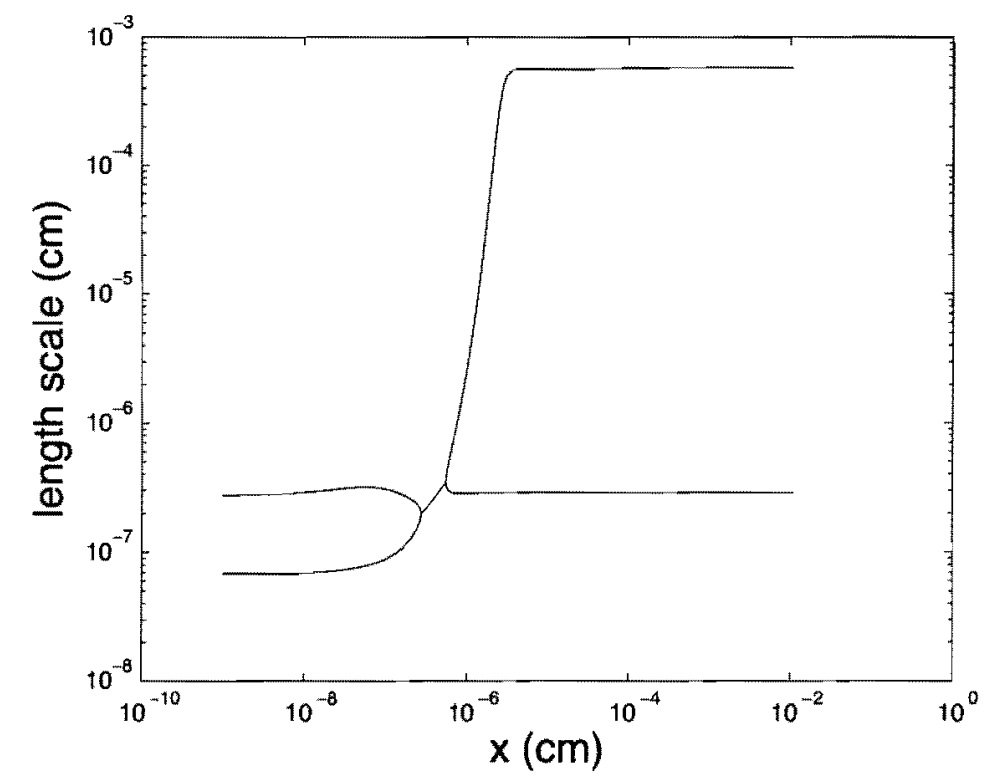

Figure 1. Reaction length scales versus distance in steady strongly overdriven ozone detonation.

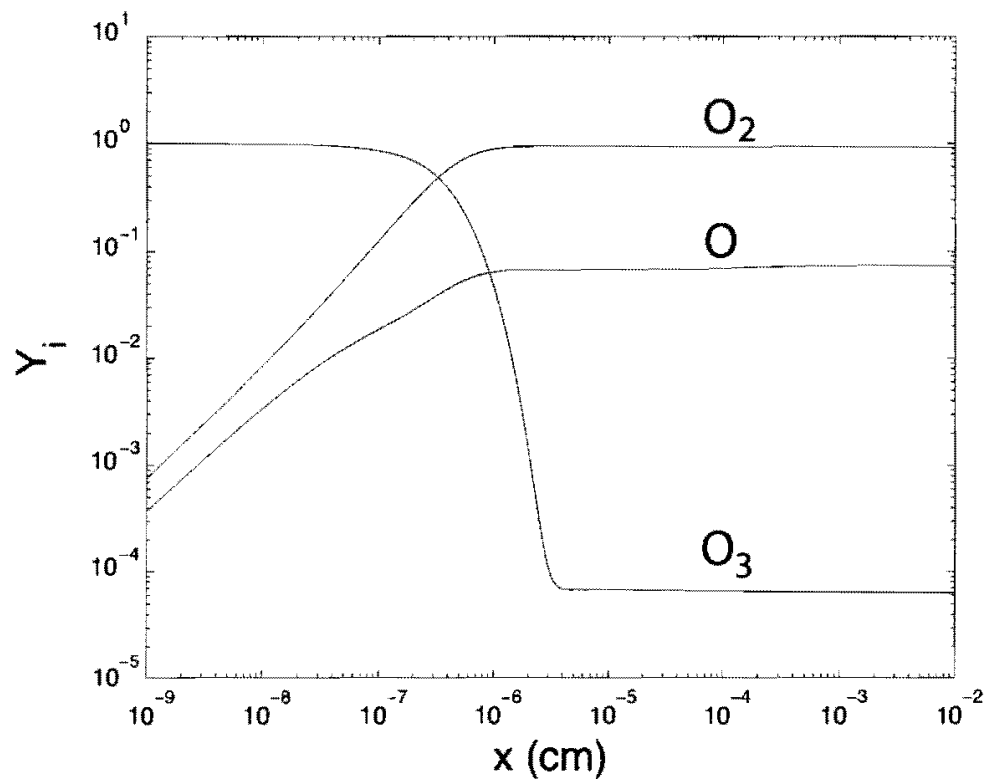

Figure 2. Species mass fraction versus distance in steady strongly overdriven ozone detonation. 


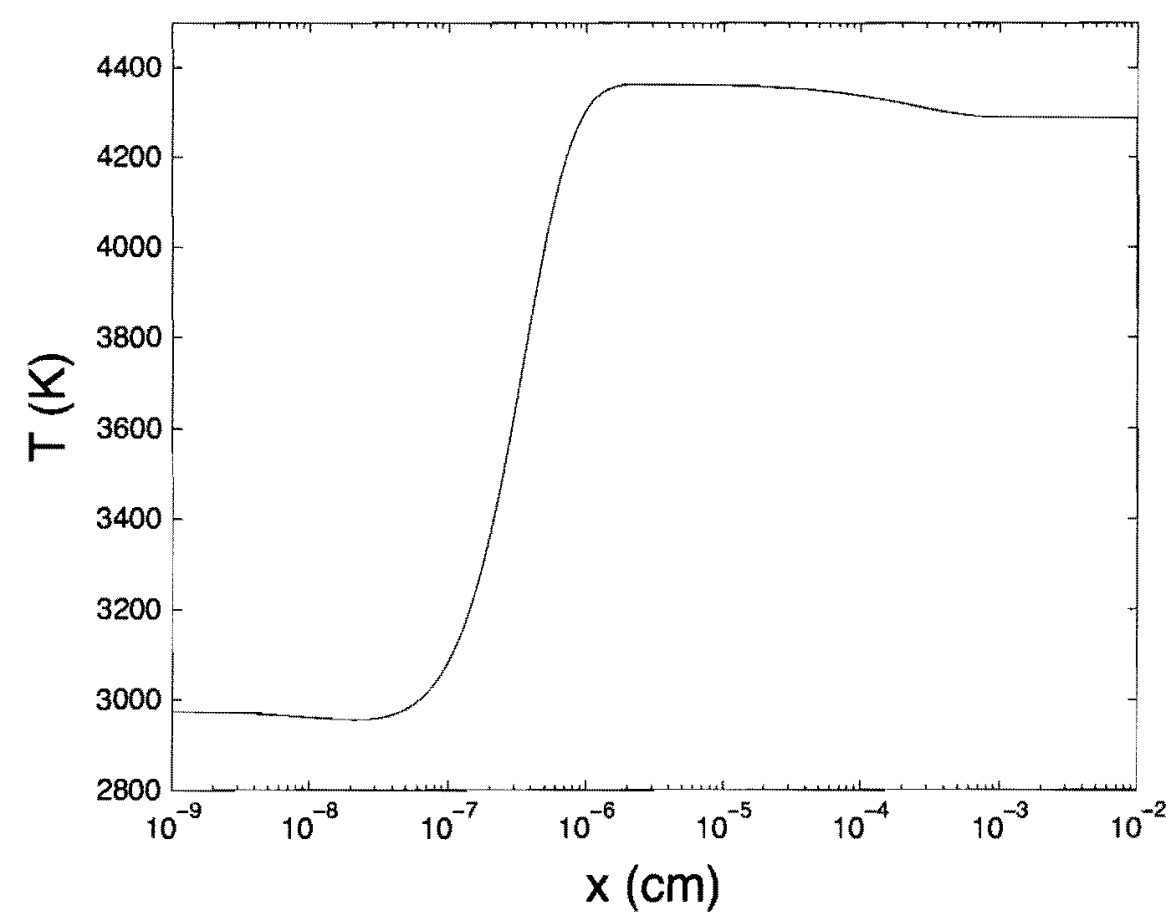

Figure 3. Temperature versus distance in steady strongly overdriven ozone detonation.

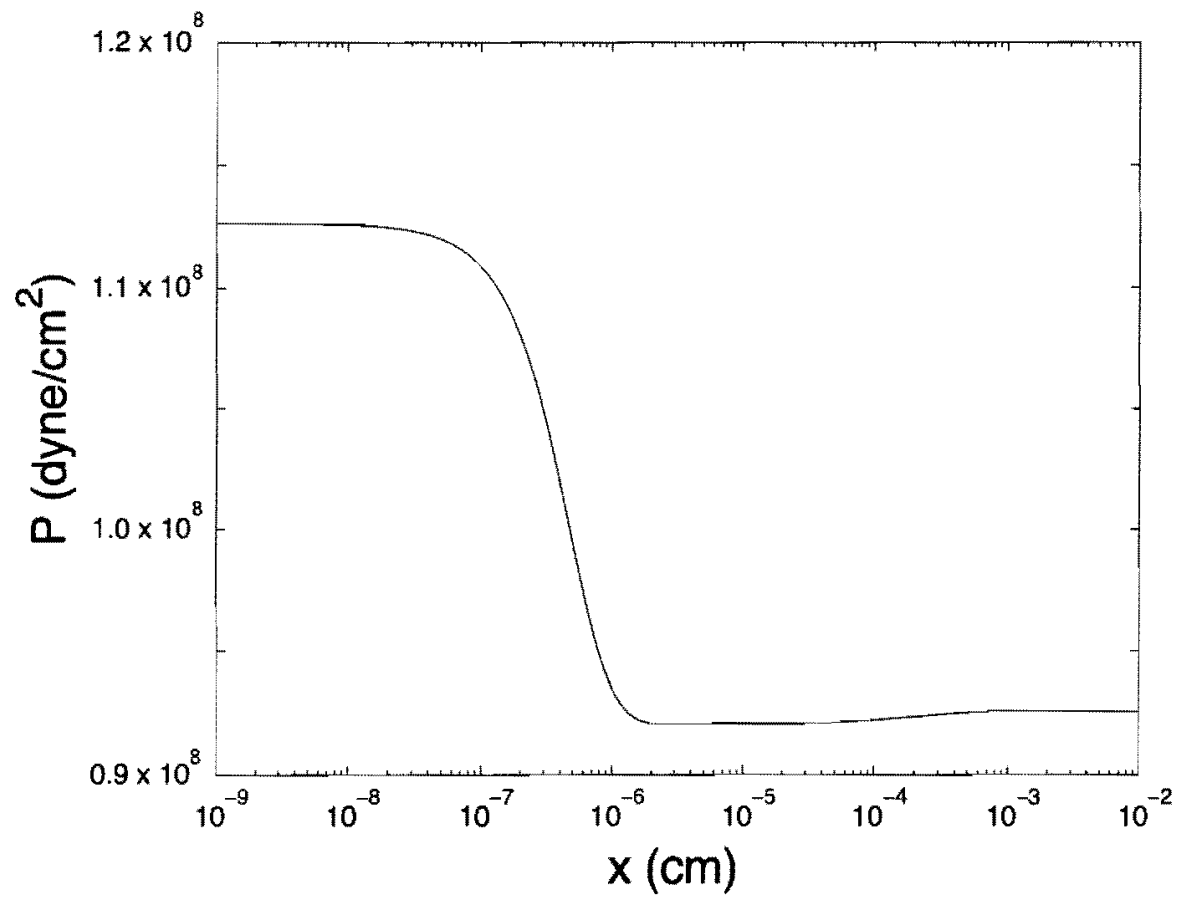

Figure 4. Pressure versus distance in steady strongly overdriven ozone detonation. 


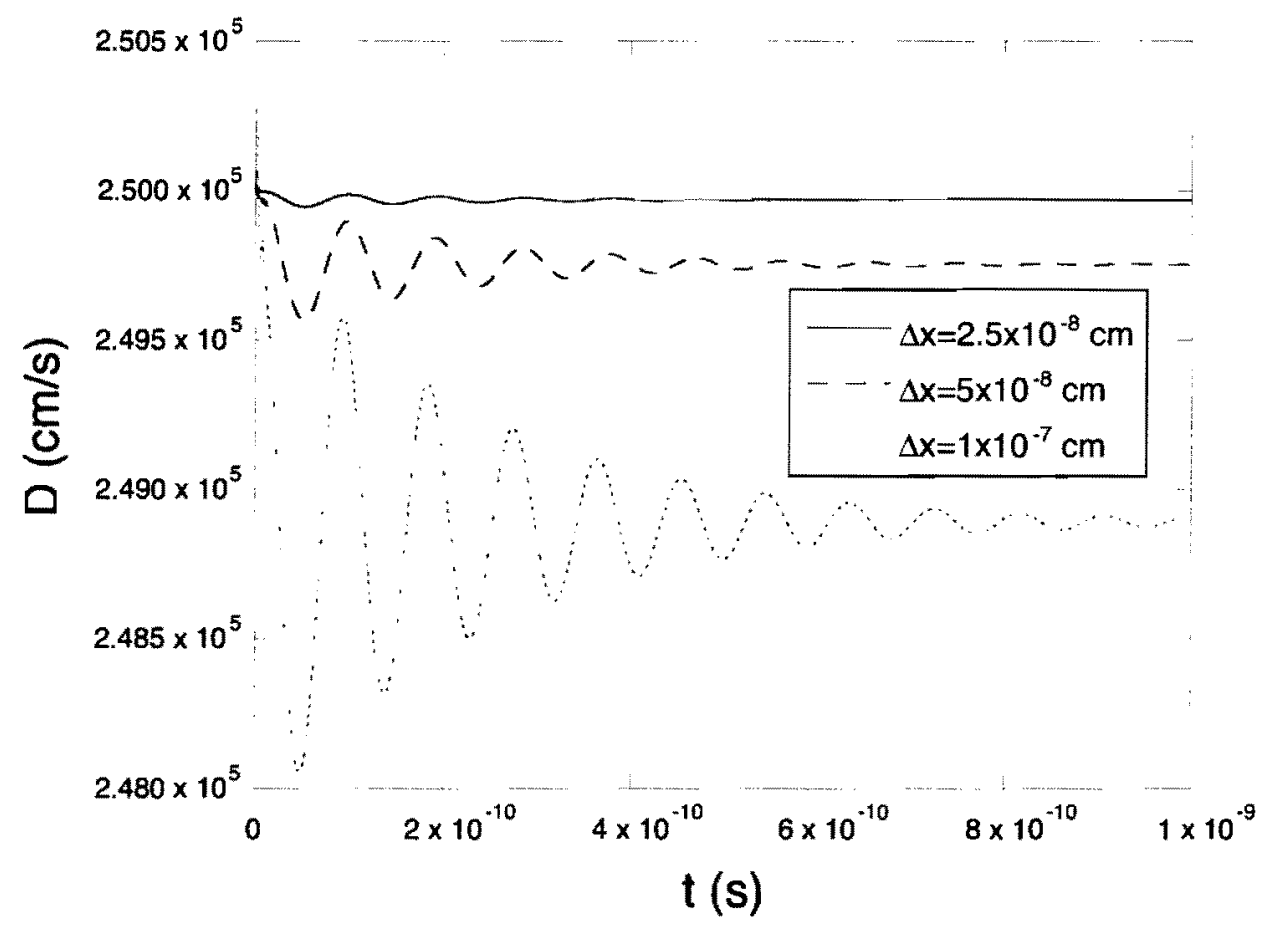

Figure 5. Computed shock velocity versus time in unsteady strongly overdriven ozone detonation. 


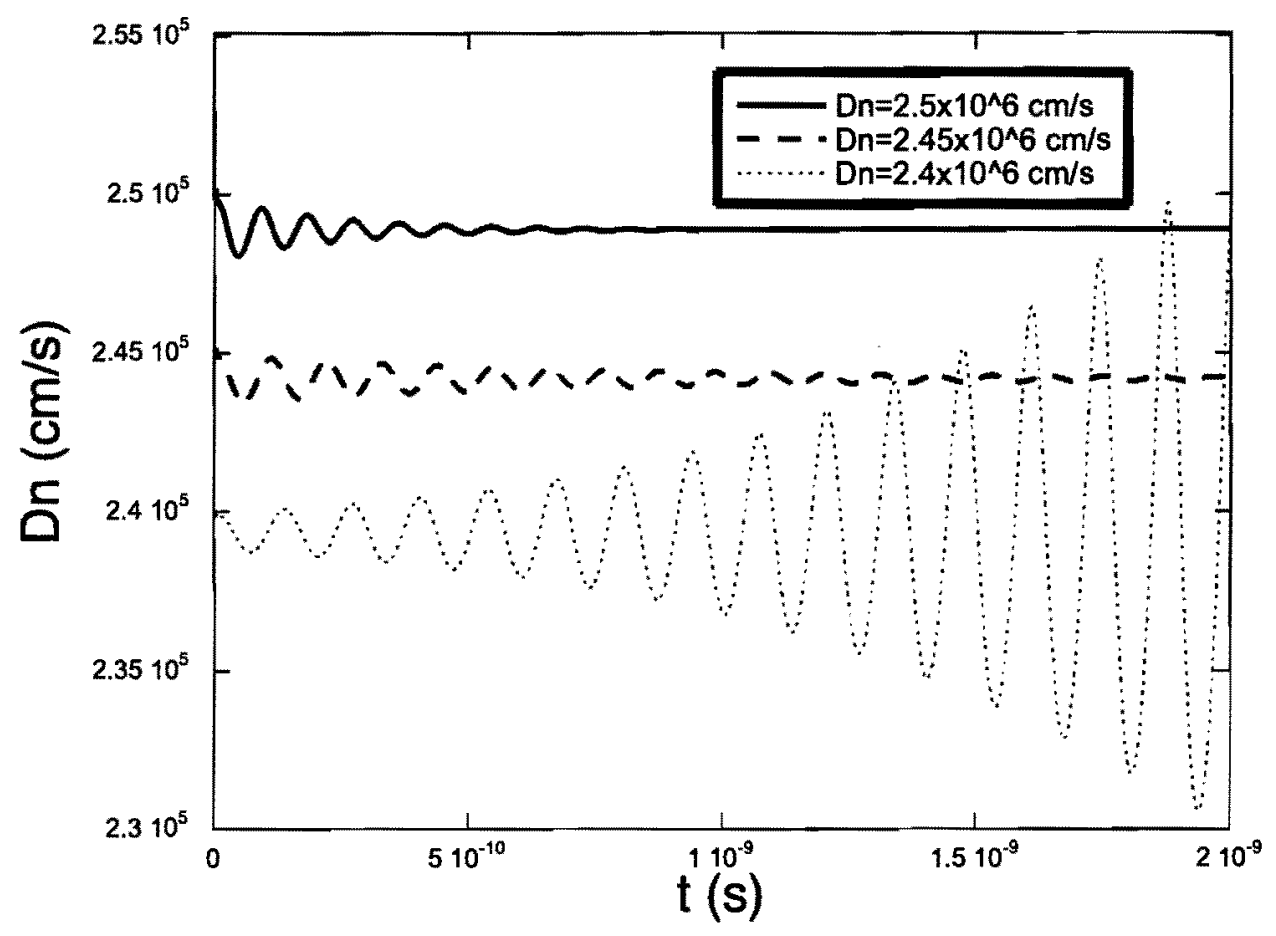

Figure 6. Detonation velocity versus time for three different initial overdrives. 


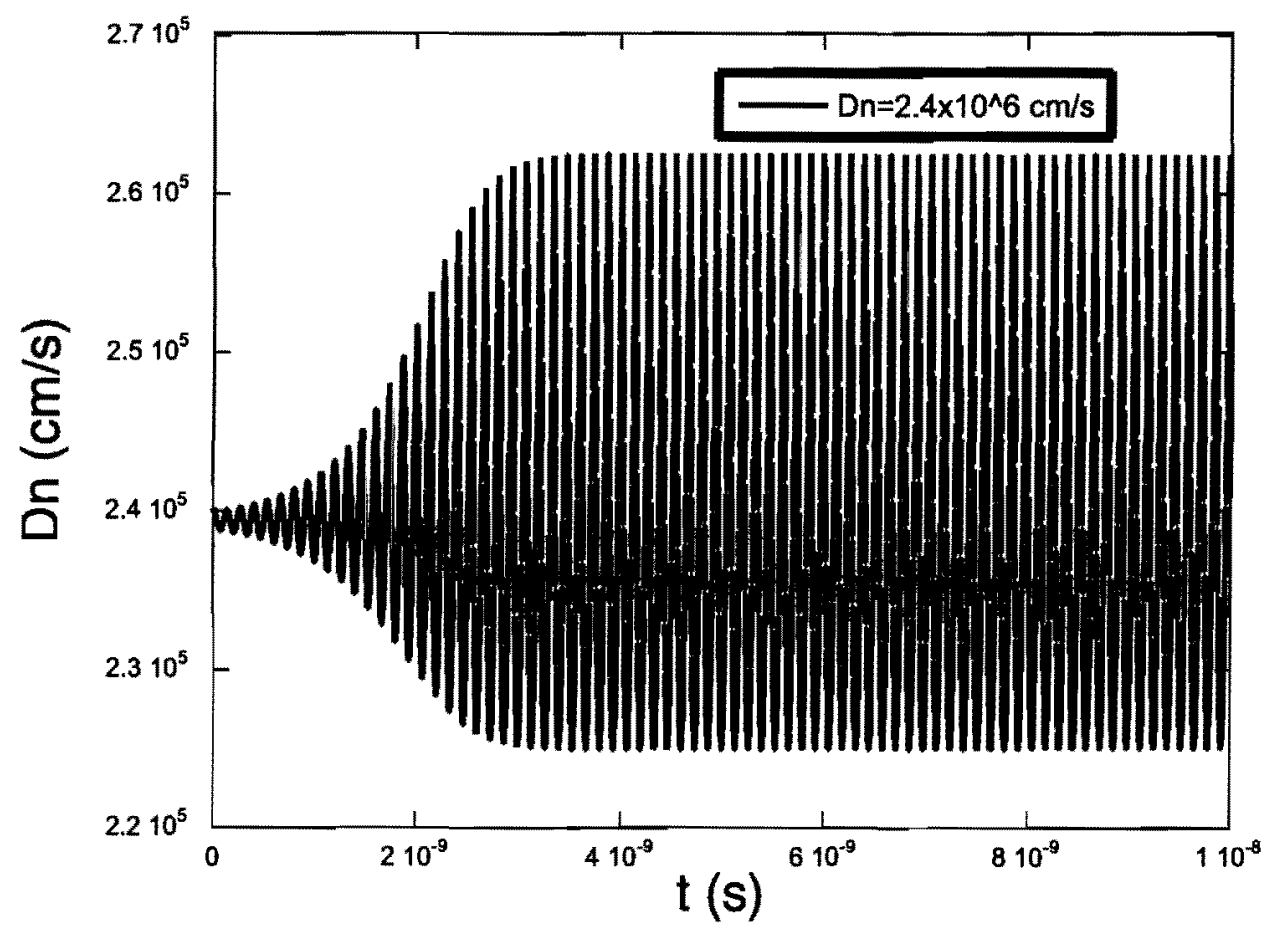

Figure 7. Long time behavior of detonation velocity for an initial overdrive corresponding to $D=2.4 \times 10^{5} \mathrm{~cm} / \mathrm{s}$ 


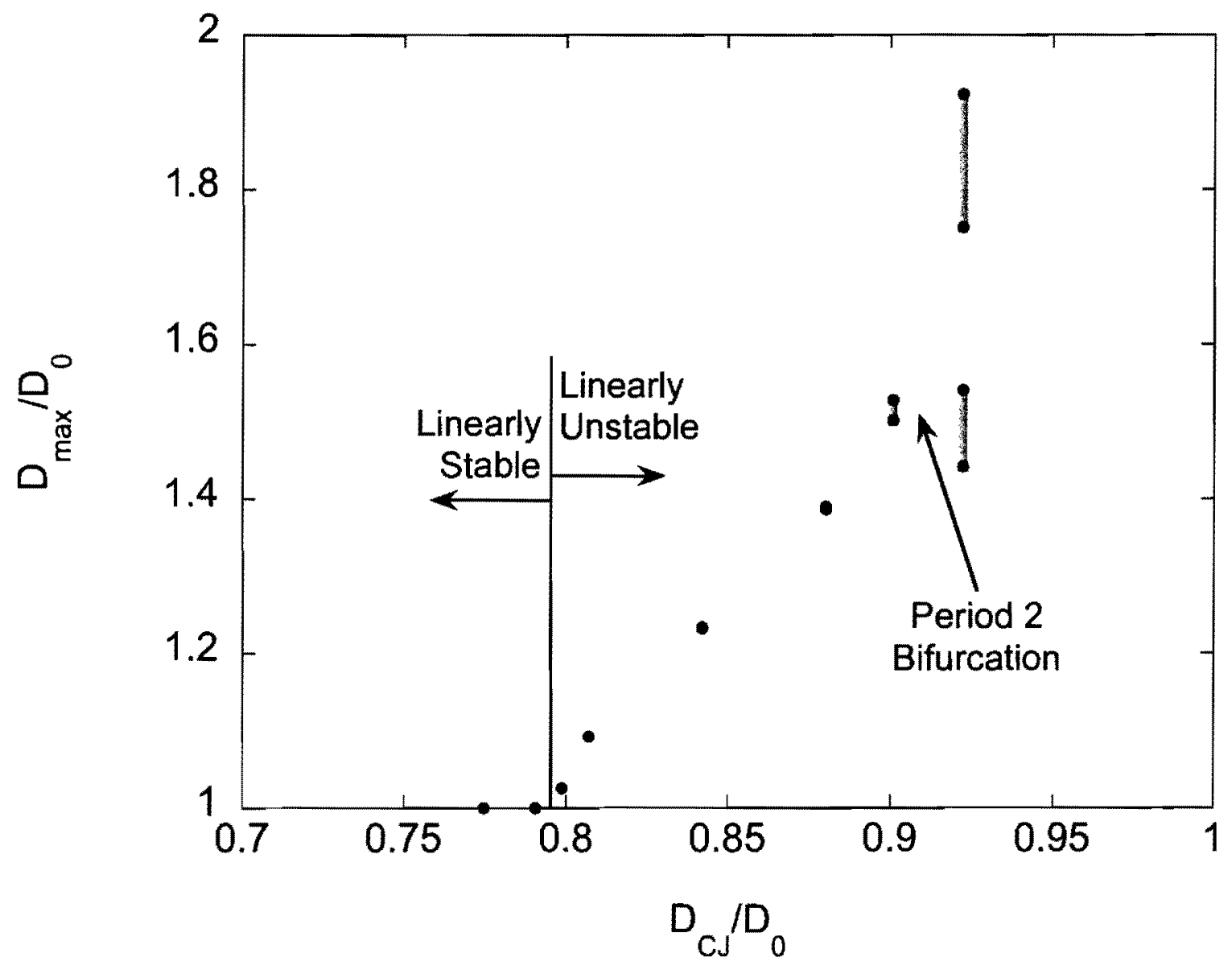

Figure 8, Bifurcation diagram showing transition to period 1 and 2 limit cycle behavior. 